\title{
Long non-coding RNA CCAT1 promotes gallbladder cancer development via negative modulation of miRNA-218-5p
}

\author{
M-Z Ma ${ }^{1,2,4}$, B-F Chu ${ }^{1,4}$, Y Zhang ${ }^{3,4}$, M-Z Weng ${ }^{1}$, Y-Y Qin ${ }^{1}$, W Gong ${ }^{1}$ and Z-W Quan ${ }^{\star, 1}$
}

Protein-coding genes account for only $\sim 2 \%$ of the human genome, whereas the vast majority of transcripts are non-coding RNAs (ncRNAs) including long ncRNAs (IncRNAs). A growing volume of literature has proposed that IncRNAs are important factors in cancer. Colon cancer-associated transcript-1 (CCAT1), an IncRNA, which was first identified in colon cancer, was previously shown to promote tumor development and be a negative prognostic factor in gastric cancer. However, the mechanism through which CCAT1 exerts its oncogenic activity remains largely unknown. Recently, a novel regulatory mechanism has been proposed in which RNAs can cross-talk with each other via competing shared for microRNAs (miRNAs). The proposed competitive endogenous RNAs could mediate the bioavailability of miRNAs on their targets, thus imposing another level of posttranscriptional regulation. In this study, we demonstrated that CCAT1 was upregulated in gallbladder cancer (GBC) tissues. CCAT1 silencing downregulated, whereas CCAT1 overexpression enhanced the expression of miRNA-218-5p target gene Bmi1 through competitively 'spongeing' miRNA-218-5p. Our data revealed that CCAT1 knockdown impaired the proliferation and invasiveness of GBC cells, at least in part through affecting miRNA-218-5p-mediated regulation of Bmi1. Moreover, CCAT1 transcript level was correlated with Bmi1 mRNA level in GBC tissues. Together, these results suggest that CCAT1 is a driver of malignancy, which acts in part through 'spongeing' miRNA-218-5p.

Cell Death and Disease (2015) 6, e1583; doi:10.1038/cddis.2014.541; published online 8 January 2015

Gallbladder cancer (GBC) is the common biliary tract cancer and the fifth most common gastrointestinal malignancy. ${ }^{1}$ Although tremendous efforts have been put into clarifying the pathophysiological mechanisms contributing to gallbladder carcinoma, much of it remains largely unknown. ${ }^{2,3}$ Therefore, it is vital to reveal the molecular mechanisms of the progression of $\mathrm{GBC}$ for the development of effective therapies.

The genome-sequencing projects revealed that the human genome comprises $<2 \%$ protein-coding genes and $>90 \%$ of the genome is transcribed as non-coding RNAs (ncRNAs). ${ }^{4}$ The ncRNA class now comprises a vast diversity of subclasses organized based on their size, structure, function and conservation. microRNAs (miRNAs), a class of short ncRNA molecules that range in size from 19 to 25 nucleotides (nt), have been one of the well-characterized classes of ncRNAs in GBC. ${ }^{5-7}$ Unlike the miRNAs, long ncRNAs (IncRNAs) are by definition > $200 \mathrm{nt}$ in length. A growing volume of literature has proposed that IncRNAs have an important role in cancer. ${ }^{8-10}$ Although much of the focus in ncRNA research (such as miRNAs and IncRNAs) has been on the regulation of protein-coding genes mediated by them, it has been suggested that ncRNAs could form a well-orchestrated regulatory interaction network: miRNAs and IncRNAs could interact with each other, imposing an additional level of posttranscriptional regulation. ${ }^{11-14} \mathrm{An}$ example of this type of regulation is exemplified by IncRNA GAS5, which binds miRNA-21 and forms a regulatory interaction. ${ }^{12}$

Colon cancer-associated transcript-1 (CCAT1), a 2628-bp IncRNA that maps to chromosome 8q24.21, was first found to be upregulated in colon cancer. ${ }^{15}$ Previous studies suggest that CCAT1 is upregulated in gastric carcinoma tissues and colon carcinoma tissues compared with adjacent normal tissues, and can be activated by c-Myc. ${ }^{8,15}$ Moreover, abnormally expressed CCAT1 promotes cell proliferation and migration in gastric cancer cells. ${ }^{8}$ However, although CCAT1 has a vital role in cancer, little is known about the mechanism through which CCAT1 exerts its oncogenic activity, and the interaction between CCAT1 and miRNAs remains largely unknown.

In the present study, we demonstrated that CCAT1 was upregulated in GBC tissues. CCAT1 upregulated the miRNA-218-5p target gene Bmi1 by competitively 'spongeing' miRNA-218-5p and then promoted the proliferation and invasiveness of GBC cells. CCAT1 may function as a part of the 'competitive endogenous RNA (ceRNA)' network. ${ }^{16}$

\footnotetext{
${ }^{1}$ Department of General Surgery, Xinhua Hospital, Shanghai Jiaotong University School of Medicine, Shanghai, China; ${ }^{2}$ Department of General Surgery, Taixing People's Hospital, Yangzhou University School of Medicine, Jiangsu Province, Taixing, China and ${ }^{3}$ Department of Gastroenterology, Yijishan Hospital Affiliated to Wannan Medical College, Wuhu, Anhui, China

${ }^{*}$ Corresponding author: Z-W Quan, Department of General Surgery, Xinhua Hospital, Shanghai Jiaotong University School of Medicine, 1665 Kongjiang Road, Shanghai 200092, China. Tel: +86 13 901647399; Fax: +86 21 25078999; E-mail: zhiwquan@163.com

${ }^{4}$ These authors are the co-first authors.

Abbreviations: ceRNA, competitive endogenous RNA; GBC, gallbladder cancer; ncRNA, non-coding RNA; miRNA, microRNA; nt, nucleotide; CCAT1, colon cancerassociated transcript-1; FBS, fetal bovine serum; RISC, RNA-induced silencing complex

Received 21.7.14; revised 12.9.14; accepted 13.11.14; Edited by G Calin
} 


\section{Results}

CCAT1 was upregulated in GBC. The relative expression level of CCAT1 was evaluated by using real-time PCR in 40 patients with GBC and paired normal tissues. CCAT1 was upregulated in GBC tissues compared with adjacent normal tissues (Figure 1a, $P<0.001)$. Furthermore, 26/40 (65\%) tumor tissues expressed 1.5-fold higher CCAT1 than normal tissues. CCAT1 was more highly expressed in tumors extending beyond the gallbladder (T3+T4) compared with tumors only detected in the gallbladder $(\mathrm{T} 1+\mathrm{T} 2)$ (Figure $1 \mathrm{~b})$, and more highly expressed in tumors spread to lymph nodes (N1/2) compared with tumors localized only in the gallbladder (N0) (Figure 1c). In order to further understand the significance of CCAT1 overexpression in GBC, we determined the potential associations between CCAT1 expression and patients' clinicopathological features. According to the median ratio of relative CCAT1 expression (3.682) in tumor tissues, the $40 \mathrm{GBC}$ patients were classified into two groups: high-CCAT1 group $(n=13)$ : CCAT1 expression ratio $\geq$ median ratio; and low-CCAT1 group $(n=27)$ : CCAT1 expression ratio $\leq$ median ratio. The detailed relationships between CCAT1 expression status and clinicopathological variables of 40 patients were summarized in Supplementary Table 2. It is worth noting that high CCAT1 expression was significantly correlated with tumor status $(P=0.029)$, lymph node invasion $(P=0.018)$ and advanced tumor node metastasis (TNM) stage $(P=0.023)$. However, CCAT1 expression level was not correlated with other parameters such as patient's gender and age (Supplementary Table 2).

We used 5'- and 3'-rapid amplification of cDNA ends RACE to map the exact sequence of CCAT1 in the cell lines used in this study. The RACE results identified two isoforms of CCAT1 (Supplementary Table 3). Next, we examined the expression level of CCAT1 in a series of GBC cell lines. H69 cell line, was used as a representative of non-tumorigenic biliary epithelial cells. Among the four invasive cell lines, the CCAT1 levels were increased as compared with that in the H69 cells (Figure 1d). We selected GBC cell line NOZ for CCAT1 knockdown and cancer cell line GBC-SD for CCAT1 overexpression, as they harbored the highest and lowest expression levels of CCAT1, respectively.

Identification of potential CCAT1-targeting miRNAs. The function of IncRNAs in human cancer may have to do with their ability to regulate protein expression. ${ }^{9,10,17}$ For example, LncRNA-Dreh could combine with the intermediate filament protein vimentin and repress its expression. ${ }^{17} \mathrm{~A}$ growing volume of literature has proposed that ncRNAs may participate in the ceRNAs regulatory network. ${ }^{11-14}$ a

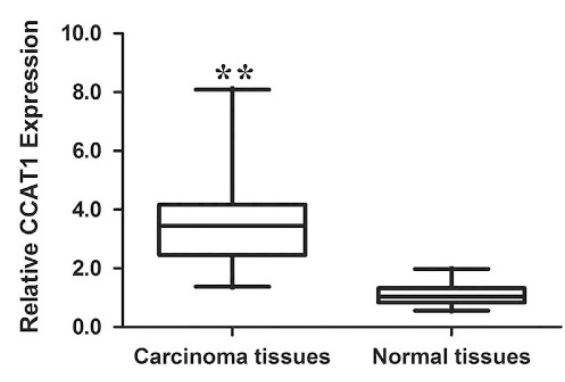

d

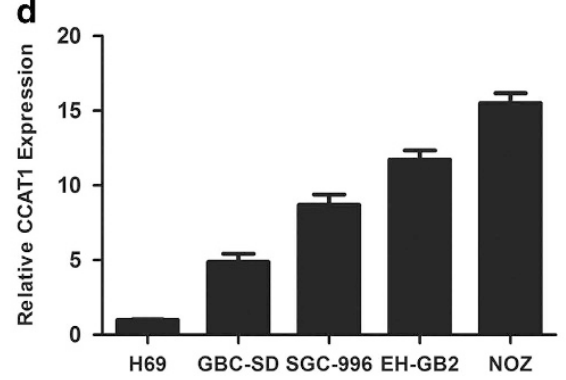

b

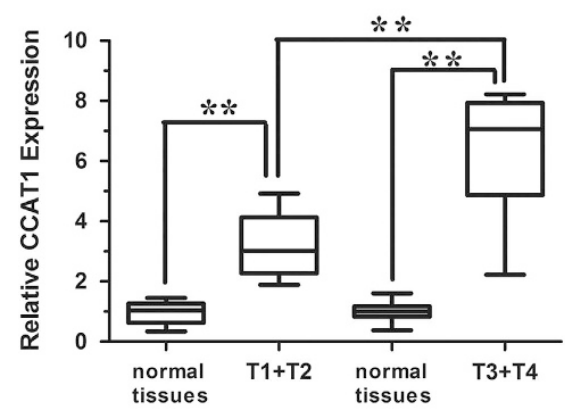

e

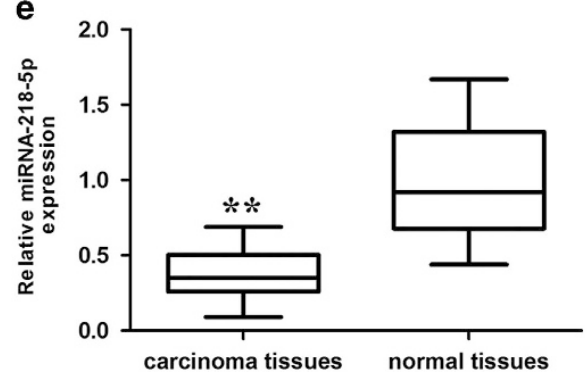

C

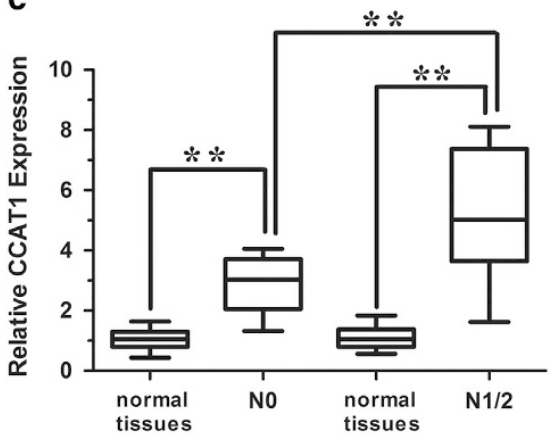

Figure 1 Expression levels of CCAT1 and miRNA-218-5p in GBC and its clinical significance. (a) Difference in expression levels of CCAT1 between GBC tissues and matched non-tumor gallbladder tissues. The expression of CCAT1 was normalized to GADPH. The statistical differences between samples were analyzed with paired samples $t$-test $(n=40, P<0.0001)$. (b) Relationship between CCAT1 expression and primary tumor growth $(P<0.0001)$. The expression of CCAT1 in T1+T2/T3+T4 stage tumors was normalized to correspondingly paired normal tissues. (c) Relationship between CCAT1 expression and lymph node metastasis $(P<0.0001)$. The expression of CCAT1 in N1+2/ N0 stage tumors was normalized to correspondingly paired normal tissues. The relative expression fold change of mRNAs was calculated by the $2^{-\Delta \Delta C t}$ method. Horizontal lines in the box plots represent the medians, the boxes represent the interquartile range and the whiskers represent the 2.5th and 97.5th percentiles. (d) Expression levels of CCAT1 in four GBC cell lines (GBC-SD, SGC-996, EH-GB2 and NOZ) and a non-tumorigenic biliary epithelial cell line (H69). The expression of CCAT1 was normalized to that in H69. The statistical differences between groups were analyzed using independent samples $t$-test. Error bars represent the mean \pm S.D. of triplicate experiments. ${ }^{*} P<0.05$; ${ }^{* \star} P<0.01$. (e) miRNA-218-5p is downregulated in GBC tissues compared with paired adjacent normal gallbladder tissues. miRNA-218-5p mRNA expression was analyzed by real-time PCR and normalized to GADPH. The relative expression fold change of mRNAs was calculated by the $2^{-\Delta \Delta C t}$ method. Horizontal lines in the box plots represent the medians, the boxes represent the interquartile range and the whiskers represent the 2.5 th and 97.5 th percentiles. The statistical differences between samples were analyzed with paired samples t-test $(n=40, P<0.0001)$ 
For example, miRNA-21 and GAS5 formed a reciprocal repression feedback loop. ${ }^{12}$ We performed a search for miRNAs that have complementary base pairing with IncRNA CCAT1, using online software program starbase v2.0 (http://starbase.sysu.edu.cn/mirLncRNA.php). ${ }^{18}$ The search results demonstrated that 22 miRNAs formed complementary base pairing with CCAT1 (Supplementary Table 4). We profiled the expression levels of the 22 miRNAs in GBC cell $\mathrm{NOZ}$ in response to CCAT1 knockdown. As demonstrated in Supplementary Table 5, there were three miRNAs that were upregulated more than 2.5-fold in response to CCAT1 inhibition. We then focused on miRNA-218-5p, which is of the greatest fold-change in response to CCAT1-specific siRNA.

The expression levels of miRNA-218-5p were also determined in GBC tissues from Figure 1. The miRNA-218-5p was markedly downregulated in GBC tissues compared with adjacent normal tissues (Figure 1e, $P<0.001$ ).

CCAT1 negatively regulated the expression of miRNA-218-5p. According to the prediction results, there was one putative binding site in exon 2 of CCAT1 (Figure 2a). To confirm the direct binding between CCAT1 and miRNA-218-5p, luciferase reporter constructs were generated. We observed that miRNA-218-5p mimics reduced the luciferase activities of wild-type (WT) CCAT1 reporter vector. However, luciferase activities in cells transfected with CCAT1 mutant and the miRNA-218-5p mimics were almost comparable to that of control cells (Figure 2b). These data confirmed the direct binding between CCAT1 and miRNA-218-5p.

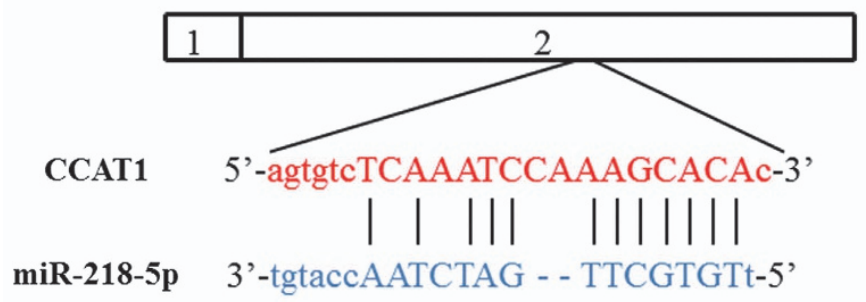

CCAT1-mt

$$
\text { 5'-AGTGTCaCgAgatCAggatgttC-3' }
$$
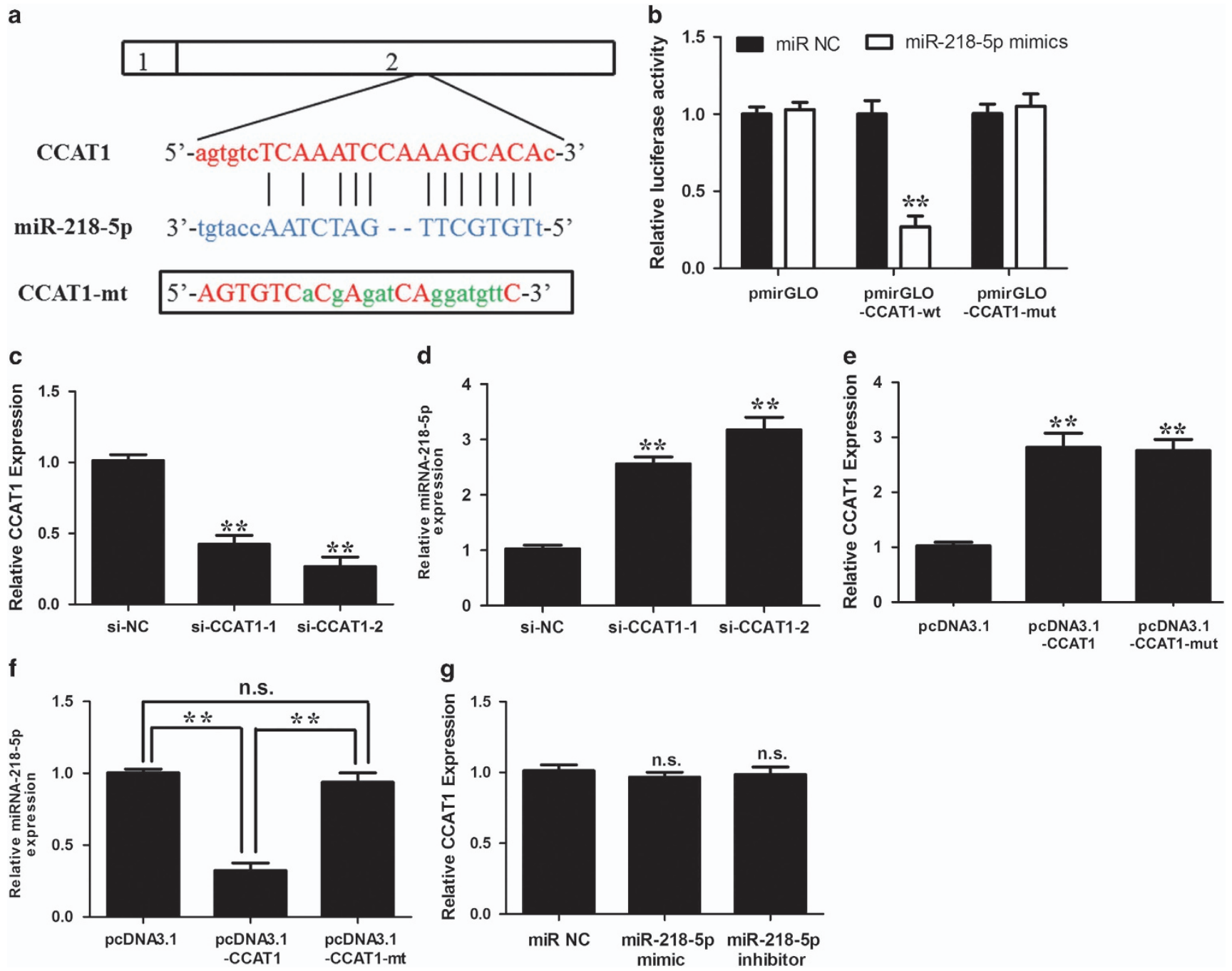

Figure 2 Identification of miRNA-218-5p as a target of CCAT1. (a) Alignment of potential CCAT1 base pairing with miRNA-218-5p as identified by Starbase v2.0 (http:// starbase.sysu.edu.cn/mirLncRNA.php). CCAT1 (top) consist of two exons, where the putative binding site is in exon 2. The mutant CCAT1 at putative binding site. (b) Luciferase activity in NOZ cells cotransfected with miRNA-218-5p mimics and luciferase reporters containing nothing, CCAT1 or mutant transcripts. Data are presented as the relative ratio of firefly luciferase activity of Renilla luciferase activity. (c) CCAT1-specific siRNA 1,2 reduced the endogenous CCAT1 mRNA level in NOZ cells. (d) Upregulation of miRNA-218-5p by si-CCAT1. NOZ cells were transfected with control siRNA or si-CCAT1-1/2, and total RNA was isolated $48 \mathrm{~h}$ after transfection. (e) CCAT1-wt or CCAT1-mut was overexpressed in GBC-SD cells. The expression level of mutant clone is similar to that of WT overexpression clone. (f) This mutant CCAT1 clone revealed no significant suppression of miRNA-218-5p compared with wild-type of CCAT1. (g) GBC-SD cells were transfected with miRNA-218-5p mimics or inhibitor, and total RNA was isolated $48 \mathrm{~h}$ after transfection. Error bars represent the mean \pm S.D. of triplicate experiments. ${ }^{*} P<0.05 ;{ }^{* *} P<0.01$; n.S., not significant 
To avoid off-target effects, we designed two CCAT1-specific siRNAs targeting different regions of CCAT1. Forty-eight hours after transfection, CCAT1 and miRNA-218-5p expression levels were measured. CCAT1 siRNAs significantly decreased the endogenous CCAT1 transcript level in NOZ cells (Figure 2c); meanwhile, CCAT1 siRNAs upregulated the expression level of miRNA-218-5p in NOZ cells (Figure 2d). In contrast, ectopic expression of CCAT1 increased the transcript level of CCAT1 in GBC-SD cells (Figure 2e), while it decreased the expression level of miRNA-218-5p (Figure 2f). We overexpressed CCAT1-wt or CCAT1-mut in GBC-SD cells (Figure $2 \mathrm{e}$ ). The expression level of mutant clone is similar to that of WT overexpression clone. This mutant CCAT1 clone revealed no significant suppression of miRNA-218-5p compared with WT of CCAT1 (Figure 2f). However, there was no obvious difference in CCAT1 level after ectopic expression or knockdown of miRNA-218-5p (Figure $2 \mathrm{~g}$ ).

The underlying mechanism of the negative regulation of miRNA-218-5p by CCAT1. To explore the underlying mechanism of such a negative regulation of miR-218 by CCAT1, we examined the expression level of pri-miRNA-218$5 p$, pre-miRNA-218-5p and mature miRNA-218-5p in response to CCAT1 knockdown. As illustrated in Figure 3a, although CCAT1 siRNA significantly upregulated mature miRNA-218-5p, it had almost no effect on the expression a

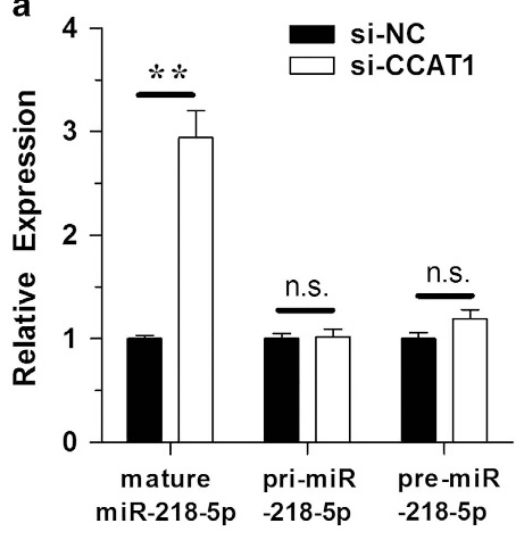

d

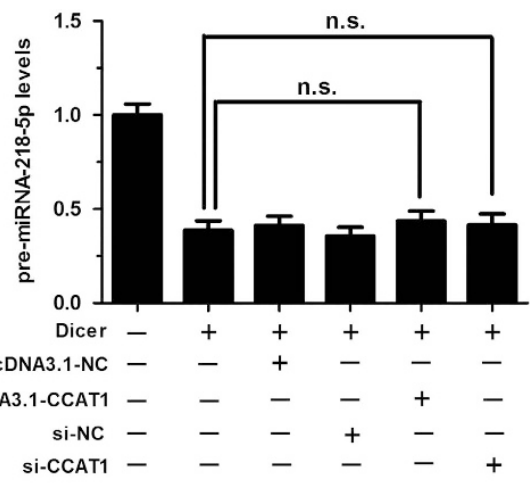

g

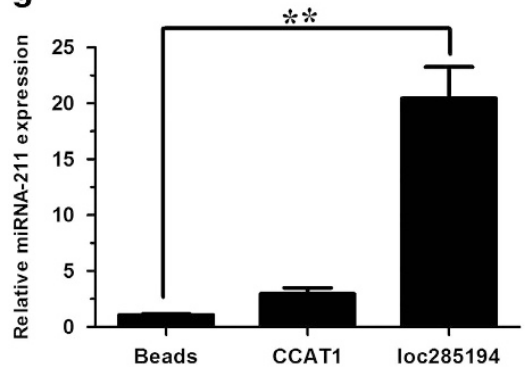

b

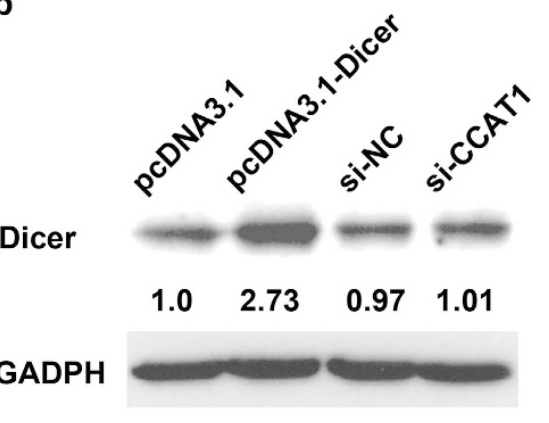

e

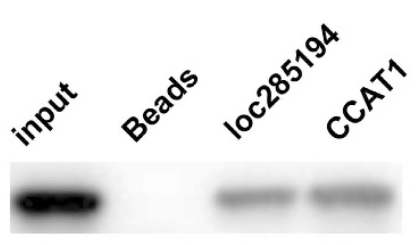

C

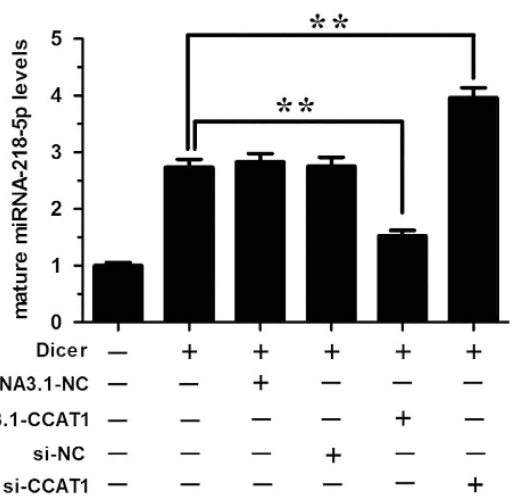

f

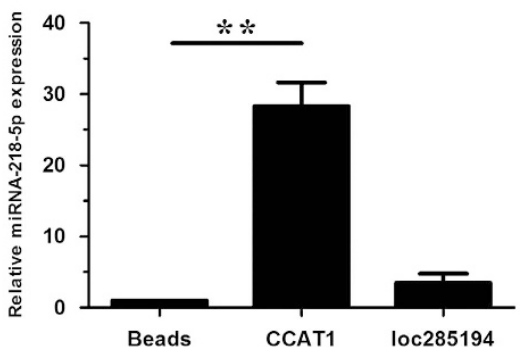

Figure 3 The underlying mechanism of the negative regulation of miRNA-218-5p by CCAT1. (a) Effect of CCAT1 on mature miRNA-218-5p, pri-miRNA-218-5p and premiRNA-218-5p. (b) NOZ cells were transfected with pcDNA3.1 or pcDNA3.1-Dicer, or si-NC or si-CCAT1 for $48 \mathrm{~h}$, and western blot analysis was performed. (c and d) NOZ cells were cotransfected with pcDNA3.1-Dicer and pcDNA3.1-CCAT1 or si-CCAT1. Forty-eight hours after transfection, cells were collected. Mature miRNA-218-5p (c) and premiRNA-218-5p (d) were analyzed by qRT-PCR. (e) Pull-down of Ago2 by biotin-labeled CCAT1 or loc285194 RNA probe, as detected by western blotting. The loc285194 lane was composed from the same gel with the same contrast. Empty vector (Beads) was used as a negative control. Loc285194 was used as a positive control. (f and $\mathbf{g}$ ) RIP followed by microRNA qRT-PCR to detect microRNAs endogenously associated with CCAT1 and loc285194. The expression level of miRNA was normalized to that in empty vector (Beads). Error bars represent the mean \pm S.D. of triplicate experiments. ${ }^{*} P<0.05 ;{ }^{*} P<0.01$, n.s., not significant 
levels of pri-miRNA-218-5p and pre-miRNA-218-5p. The data suggest that this negative regulation effect might be through a posttranscriptional mechanism. Mature miRNAs are generated through a two-step processing by Drosha and Dicer. ${ }^{19}$ The initial process occurs in the nucleus that Drosha-DGCR8 complex cleaves pri-miRNA into pre-miRNA. The second processing step occurs in the cytoplasm when pre-miRNA was cleaved by Dicer complex to create a mature miRNA duplex. $^{20}$ To further explore whether the downregulation of mature miRNA-218-5p by CCAT1 was through the interference of the processing of Dicer, we applied Dicer to assay the levels of pre-miRNA-218-5p and mature miRNA-218-5p. Ectopic expression of Dicer significantly upregulated the protein level of Dicer (Figure 3b). What's more, CCAT1 had no significant effect on the expression level of Dicer (Figure $3 b$ ). Our results demonstrated that although Dicer overexpression increased the level of mature miRNA-218-5p (Figure $3 c$ ) and in the meantime decreased the level of premiRNA-218-5p (Figure 3d), CCAT1 overexpression inhibited the increase of mature miRNA-218-5p induced by Dicer, and CCAT1 silencing had an opposite effect (Figure 3c). Both CCAT1 overexpression and silencing had no significant effect on the expression level of pre-miRNA-218-5p with Dicer overexpression (Figure $3 d$ ). Taken together, it suggests that the regulation of miRNA-218-5p by CCAT1 is through another mechanism.

Previous studies revealed that miRNAs are present in the cytoplasm in the form of miRNA ribonucleoprotein complexes that also contain Ago2, the core component of the RNAinduced silencing complex (RISC). miRNAs exert their genesilencing functions through RISC. ${ }^{21}$ Potential miRNA targets can be isolated from this complex after Ago2 coimmunoprecipitation, ${ }^{11,22}$ as Ago2 is a vital component of RISC complex necessary for siRNA or miRNA-mediated gene silencing. Furthermore, CCAT1 is shown to be a cytoplasmlocated IncRNA. ${ }^{23}$

We performed RNA pull-down experiments by using CCAT1 probe and then examined Ago2 and miRNA-218-5p simultaneously as described previously, ${ }^{11,12}$ to determine whether CCAT1 and miRNA-218-5p are in the same RISC complex. Loc285194 (Liu et al. ${ }^{11}$ ) was used as a positive control. A biotin-labeled CCAT1 RNA probe was synthesized and mixed with the cellular extract. After pull-down experiment with streptavidin beads, RNA or protein that interacts with the probe is expected to co-precipitate with the biotin-labeled probe. The in vitro RNA pull-down experiment was performed to confirm the direct physical association between CCAT1 and Ago2. As a result, we detected Ago2 (Figure 3e). Furthermore, we detected miRNA-218-5p in the same pellet, supporting that miRNA-218-5p is bona fide CCAT1-targeting miRNA (Figure 3f). To demonstrate the specificity of the association between CCAT1 and miRNA-218-5p, we detected miRNA-211, which was shown to be negatively regulated by loc285194 (Liu et al. ${ }^{11}$ ) and did not form complementary base pairing with CCAT1 according to our prediction results. Quantitative reverse-transcription PCR (qRT-PCR) analysis revealed that loc285194 was significantly enriched for miRNA-211 compared with the empty vector (Beads) and CCAT1 (Figure $3 \mathrm{~g}$ ). Our data suggest that the regulation between miRNA-218-5p and CCAT1 might be in a way similar to the miRNA-mediated silencing of protein-coding genes. ${ }^{11,14}$

CCAT1 negatively regulates $B m i 1$, a miRNA-218-5p target gene. Having demonstrated that CCAT1 could negatively regulate miRNA-218-5p expression, we then examined the functional aspect. miRNA-218-5p was shown to be downregulated in a variety of carcinomas, including mesenchymal glioblastoma, ${ }^{24}$ pancreatic cancer $^{25}$ and renal cell carcinoma, ${ }^{26}$ and exhibits tumor-suppressive activities, ${ }^{24-27}$ while CCAT1 was shown to promote tumor progression. ${ }^{8}$ In the first place, we examined whether CCAT1 has any effect on miRNA-218-5p target genes and found that CCAT1 knockdown inhibited the expression of Bmi1 (Tu et al. ${ }^{27}$ ) and caveolin-2 (Yamasaki et al. ${ }^{26}$ ), whereas co-transfection of miRNA-218-5p inhibitor attenuated this inhibition (Figure 4a). The incomplete rescue of caveolin-2 by miRNA-218-5p inhibitor suggests that other mechanisms might also be involved in its regulation. Previous studies suggest that miRNA-218-5p inhibits tumor invasion, migration and proliferation by targeting the polycomb group gene Bmi1 (Tu et al. ${ }^{27}$ ). As CCAT1 shares regulatory miRNA-218-5p with Bmi1 (Figure 4b), we would like to explore whether CCAT1 could modulate miRNA-218-5p and then Bmi1. We observed that although si-CCAT1 decreased Bmi1 transcript and protein levels, miRNA-218-5p inhibitor abrogated this decrease in NOZ cells (Figures $4 \mathrm{c}$ and $d$ ). Similar phenomenon was observed in GBC-SD cells (Figure 4e). In GBC-SD cells, although ectopic expression of CCAT1 upregulated Bmi1 at both transcript and protein levels, miRNA-218-5p mimic relieved the increase (Figures $4 \mathrm{f}$ and $\mathrm{g}$ ). Yet, CCAT1mut had no significant effect on the expression of Bmi1 (Figure 4g).

Our results revealed that CCAT1 and Bmi1 shared the same miRNA-responsive element in their sequences and displayed the same miRNA-218-5p-dependent regulation pattern. To explore whether the observed effects depends on the regulation of the $3^{\prime}$ untranslated regions ( $3^{\prime}-\mathrm{UTR}$ ) of Bmi1, a luciferase reporter plasmid containing the $3^{\prime}$-UTR of Bmi1 was constructed. Luciferase plasmid was transfected into NOZ and GBC-SD cells. As illustrated in Figure 5a, CCAT1 knockdown decreased the luciferase activity in the Luc-Bmi1-3'-UTRtransfected NOZ cells, which was rescued by miRNA-218-5p inhibitor. On the other hand, ectopic expression of CCAT1 WT, but not CCAT1-mut, increased the luciferase activity in the Luc-Bmi1-3'-UTR-transfected GBC-SD cells. However, miRNA-218-5p mimics abolished this upregulation (Figure $5 b$ ). Furthermore, CCAT1 transcript level was significantly correlated with miRNA-218-5p mRNA level in GBC tissues (Figure 5c).

\section{CCAT1's oncogenic activity is in part through negative} regulation of miRNA-218-5p and then modulating Bmi1 in vitro. To investigate the biological association between CCAT1 and miRNA-218-5p, we employed gain- and loss-offunction approaches. Bmi1 siRNAs resulted in significant downregulation of the protein level of Bmi1 (Figure 6a). The percentage of S-phase cells and the number of invasive cells were reduced after Bmi1 knockdown (Figures 6b-e). 


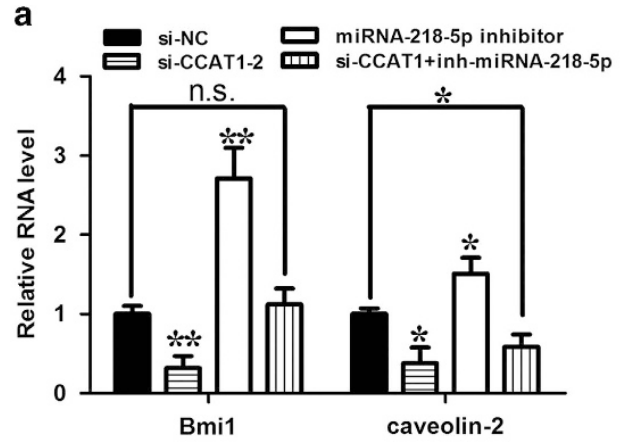

C

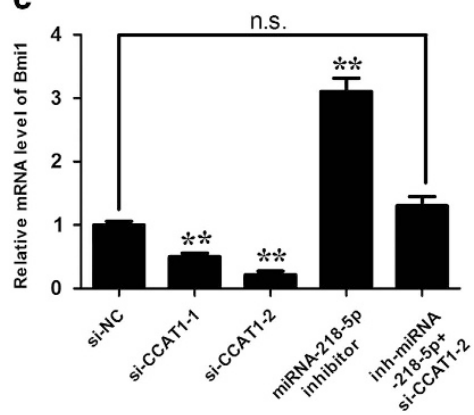

d

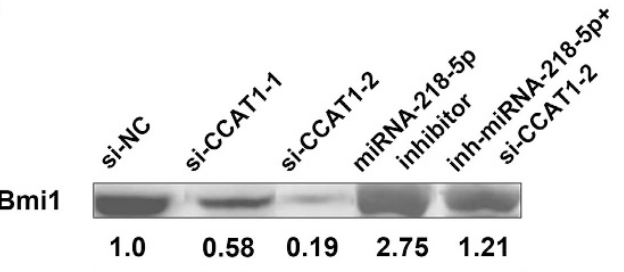

GADPH

CCAT1

miR-218-5p

Bmi1 3'UTR

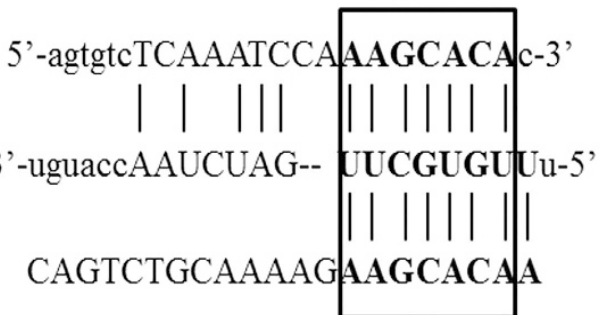

f

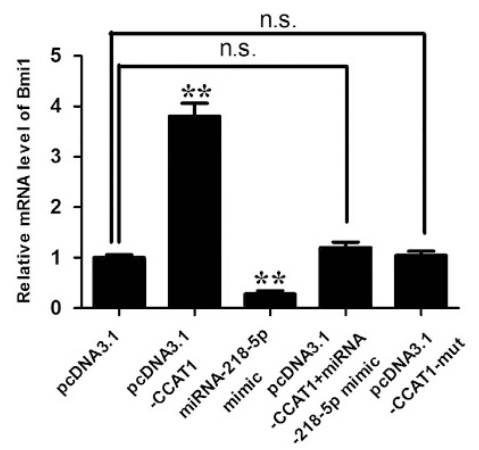

g

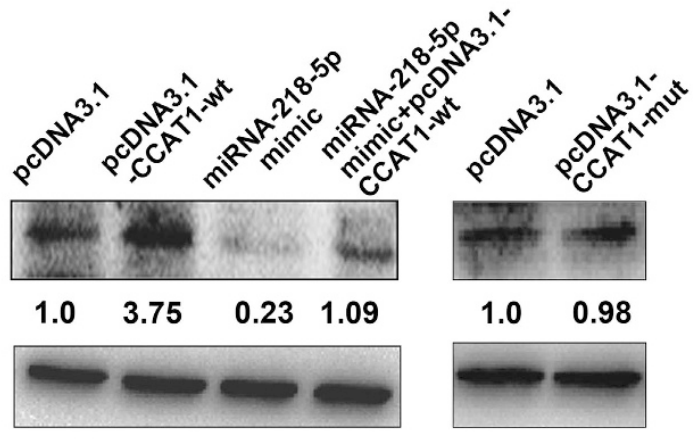

Figure 4 Regulation of Bmi1 by CCAT1. (a) NOZ cells were transfected with si-NC, si-CCAT1, si-CCAT1+miRNA-218-5p inhibitor and miRNA-218-5p inhibitor. qRT-PCR was performed $48 \mathrm{~h}$ post transfection. (b) Nucleotide resolution of miRNA-binding sites in CCAT1 and Bmi1. As indicated, CCAT1 and Bmi1 shared the same miRNA-responsive element in their sequences. miRNA-218-5p is a validated Bmi1-targeting miRNA. The mRNA (c) or protein (d) levels of Bmi1 in NOZ cells transfected with si-NC, si-CCAT1, si-CCAT1+miRNA-218-5p inhibitor and miRNA-218-5p inhibitor. (e) The mRNA levels of Bmi1 in GBC-SD cells transfected with si-NC, si-CCAT1-2, si-CCAT1-2+miRNA-218-5p inhibitor and miRNA-218-5p inhibitor. The mRNA (f) or protein (g) levels of Bmi1 in GBC-SD cells transfected with pcDNA3.1, pcDNA3.1-CCAT1, miRNA-218-5p mimic, pcDNA3.1-CCAT1+miRNA-218-5p mimic and pcDNA3.1-CCAT1-mut. Error bars represent the mean \pm S.D. of triplicate experiments. ${ }^{*} P<0.05 ;{ }^{* *} P<0.01$

The data suggest that Bmi1 has an important role in proliferation and migration of GBC.

A decrease in the percentage of S-phase cells was observed in GBC cell NOZ after transfection of CCAT1 siRNA but was reversed by cotransfection of miRNA-218-5p inhibitor (Figures $7 a$ and b). Similar phenomenon was observed in invasion (Figures $7 \mathrm{c}$ and d) of GBC cells. All these data imply that the oncogenic activity of CCAT1 is partly associated with the regulation of miRNA-218-5p and then Bmi1.

CCAT1's oncogenic activity is in part through negative regulation of miRNA-218-5p in vivo. To provide additional evidence to the idea that CCAT1's oncogenic activity is in part through the negative regulation of miRNA-218-5p, we stably transfected NOZ cells with a lentivirus construct containing desired vector. The cells were then injected subcutaneously into nude mice. Tumor volume was measured on a weekly basis and mice were killed 4 weeks after cancer cell inoculation. Palpable tumor formed within 5 days. After 4 weeks, we observed a decrease in tumor growth in the NOZ-shRNA-CCAT1 group compared with the NOZ-vector group and miRNA-218-5p inhibitor abrogated this decrease in tumor growth (Figures $8 a$ and $b$ ). Furthermore, our data demonstrated that the expression level of Bmi1 of the tumor from the shRNA-CCAT1 group was lower compared with the control group with immunochemistry analysis and miRNA-218-5p inhibitor abrogated the decrease (Figures $8 \mathrm{c}$ and $\mathrm{d}$ ) 
a

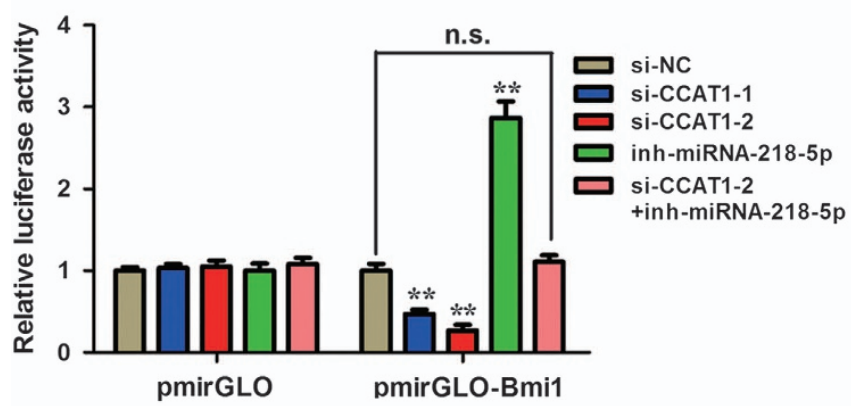

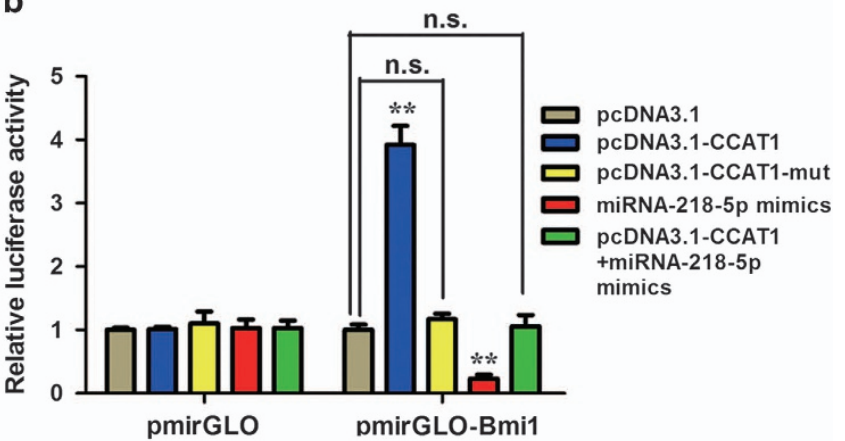

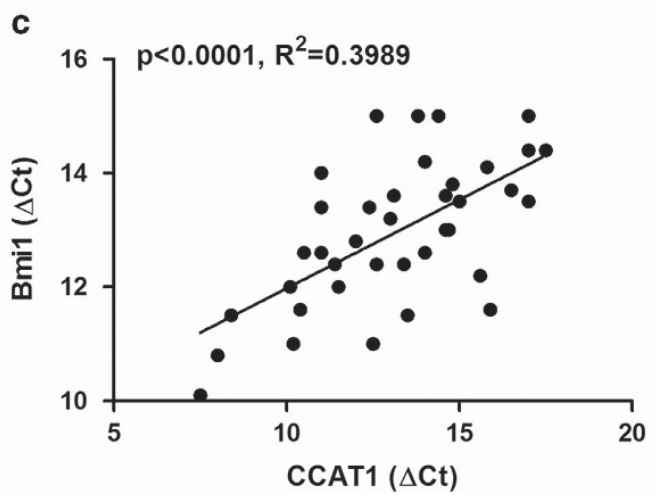

Figure 5 Regulation of Bmi1 by CCAT1 depends on the regulation of the $3^{\prime}$-UTR of Bmi1. (a and b) Luciferase activity in NOZ cells (a) transfected with luciferase reporters containing Bmi1 3'-UTR or nothing. Data are represented as the relative ratio of firefly luciferase activity to Renilla luciferase activity. Error bars represent the mean \pm S.D. of triplicate experiments. ${ }^{*} P<0.05 ;{ }^{*} P<0.01$; n.s., not significant. (c) The correlation between CCAT1 transcript level and Bmi1 mRNA level was measured in 40 gallbladder tissues. The $\triangle \mathrm{Ct}$ values (normalized to GADPH) were subjected to Pearson's correlation analysis

\section{Discussion}

Recent evidence has shown that ncRNAs have an important role in cancer pathogenesis and could provide new insights into the biology of this disease.$^{5-12}$ Over the past decade, the researches of miRNAs have dominated the field of ncRNA regulation. ${ }^{5-8}$ However, the role of IncRNAs in the tumorigenesis of GBC remains largely unknown. Understanding the precise molecular mechanism by which IncRNAs function would facilitate the development of IncRNA-directed diagnostics and therapeutics against cancers. In this study, we provide evidence that CCAT1 exhibits oncogenic activities partly through modulation of miRNA-218-5p and then Bmi1.

A growing number of reports suggests the existence of a widespread interaction network involving ceRNAs, where ncRNAs could regulate modulatory RNA by binding and titrating them off their binding sites on protein coding messengers. ${ }^{13,28}$ An example of this type of regulation is exemplified by HULC, an IncRNA highly upregulated in liver cancer, whose upregulated expression is in part to its inhibitory effects on the expression and activity of miRNA-372 (Wang et al. ${ }^{29}$ ). Similar report suggests that $\mathrm{H} 19$ acts as a molecular sponge to modulate let-7 availability and inhibits muscle differentiation through Antagonizing let-7 (Kallen et al. ${ }^{30}$ ). Han et al. ${ }^{31}$ revealed that miRNA-125b suppresses the expression of oncogenic IncRNA MALAT1. Liu et al. ${ }^{11}$ demonstrated a reciprocal repression between loc285194 and miRNA-211 in colon cancer. Similar phenomenon was observed between GAS5 and miRNA-21 (Zhang et $a l^{12}$ ). The entire spectrum of ncRNA regulatory layer, especially the interaction between IncRNA-miRNA remains to be explored.

CCAT1 is an IncRNA initially characterized in colon cancer. ${ }^{15}$ CCAT1 was found to be activated by c-Myc, and is crucial for cancer cell invasion and proliferation in gastric cancer. $^{8}$ However, the mechanism through which CCAT1 exerts its oncogenic functions remains to be explored. In this study, we would like to explore the interaction between CCAT1 and miRNAs in GBC. We first examined the expression pattern of CCAT1 in GBC tissues. CCAT1 was found to be upregulated in GBC tissues compared with adjacent normal tissues and its expression pattern was associated with tumor status and lymph node status. It is worth mentioning that although we did make efforts to collect the normal epithelial tissues as the adjacent normal tissues, as it is the normal epithelial layer in the gallbladder that gives rise to the carcinoma, it is inevitable that the 'normal' samples contain some non-epithelial tissue. Thus, it might bring some deviance to the expression levels of CCAT1 in the adjacent normal tissues. However, we performed two quality controls over the selection of adjacent normal tissues to minimize the deviances.

We performed a search for miRNAs that had complementary base pairing with IncRNA CCAT1 and identified 22 miRNAs that formed complementary base pairing with CCAT1. The expression changes of the 22 miRNAs in response to CCAT1 knockdown were examined. We focused on miRNA-218-5p, as it is of the greatest 
a

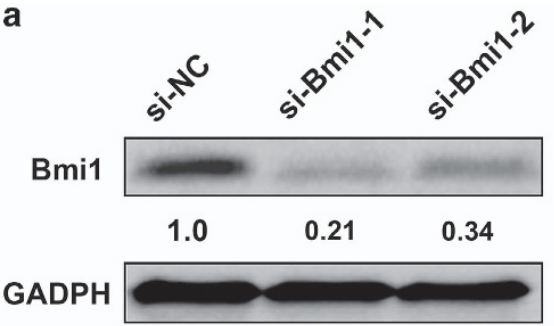

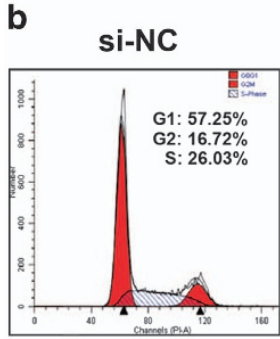

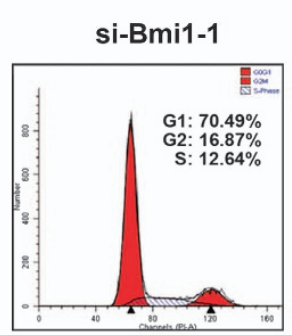

d

si-NC

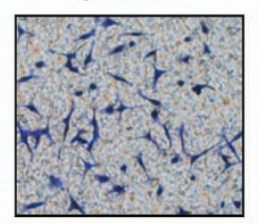

si-Bmi1-1

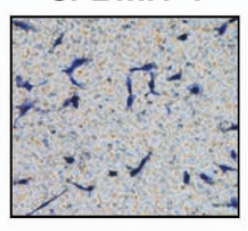

C

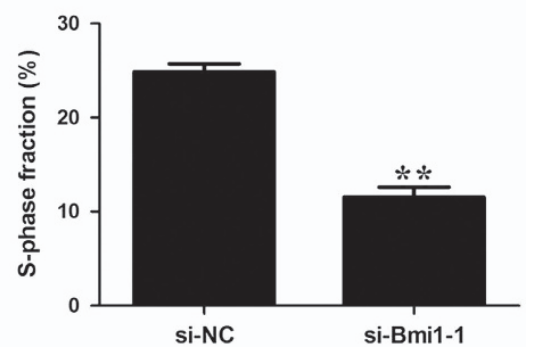

e

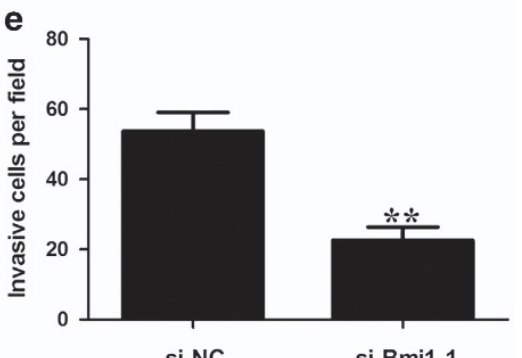

Figure 6 Bmi1 promotes the growth and invasion of GBC cells. (a) Bmi1-specific siRNA 1,2 effectively suppressed the protein level of Bmi1. (b, c) Flow cytometric analysis was performed in NOZ cells. Data were expressed as percentage distribution of cells in G0/G1, S and G2/M phases of the cell cycle. (d, e) The invasive ability of NOZ cells can be blocked by Bmi1 downregulation. Error bars represent the mean \pm S.D. of triplicate experiments. ${ }^{*} P<0.05 ;{ }^{* *} P<0.01$

fold change. Knockdown of CCAT1 increased the expression of miRNA-218-5p, while ectopic expression of CCAT1 induced the downregulation of miRNA-218-5p and the miRNA-218-5p-binding site is indispensable for the CCAT1-mediated repression.

Recent studies suggest that IncRNAs may exert functions through targeting miRNAs. $\mathrm{H} 19$ was shown to inhibit muscle differentiation through Antagonizing let-7 (Kallen et al. ${ }^{30}$ ). linc-MD1 was demonstrated to 'sponge' miR-133 and miR-133 to regulate muscle differentiation. ${ }^{32} \mathrm{~A}$ recently identified IncRNA, CHRF, was demonstrated to regulate cardiac hypertrophy by targeting miRNA-489 (Wang et al. ${ }^{33}$ ). miRNA-218-5p was demonstrated to suppress tumor growth, ${ }^{24-27}$ while CCAT1 was shown to promote tumor progression. ${ }^{8}$ We studied the biological aspect of CCAT1 and miRNA-218-5p in GBC cells. The present study revealed that although knockdown of CCAT1 inhibited the proliferation and migration of GBC cells, miRNA-218-5p inhibitor reversed the effects that CCAT1-specific siRNA exerted partially through modulating Bmi1, both in vivo and in vitro. Our study suggests that CCAT1 may promote tumor development through 'spongeing' miRNA-218-5p.

What's more, CCAT1 transcript level was found to be correlated with Bmi1 mRNA level in GBC tissues, providing additional evidence to such a regulatory network. Furthermore, we explored the underlying mechanism of the negative regulation of miRNA-218-5p by CCAT1. Our data demonstrated that CCAT1 had no effect on the expression level of primiRNA-218-5p and pre-miRNA-218-5p. CCAT1 inhibited the increase of mature miRNA-218-5p induced by Dicer overexpression and CCAT1 silencing had an opposite effect. Furthermore, CCAT1 had no significant effect on the expression level of Dicer. CCAT1 overexpression and silencing had no significant effect on the expression level of pre-miRNA-218-5p. It suggests this regulatory effect might be through another mechanism. We found that CCAT1 and miRNA-218-5p bound to the same RISC complex. With respect to the regulation of miRNA-218-5p by CCAT1, we hypothesize that CCAT1 promotes the degradation of miRNA-218-5p via binding to the RISC complex, in which case CCAT1 functions as an 'miRNA,' while miRNA-218-5p acts as the 'miRNA targeting mRNA'. The precise mechanism requires further investigation in the future.

However, the alignment between the CCAT1 and miRNA-218-5p is not very specific, as 22 miRNAs were predicted to form complementary base pairing with CCAT1. What's more, miRNA-218-5p may also act independently of IncRNA-CCAT1, as it shares homology with a number of protein-coding genes such as caveolin-2 (Yamasai et al. ${ }^{26}$ ) and Bmi1 (Tu et al. ${ }^{27}$ ).

Our study suggests another layer of regulation involving ncRNAs in both molecular and biological aspects. A better 
si-NC

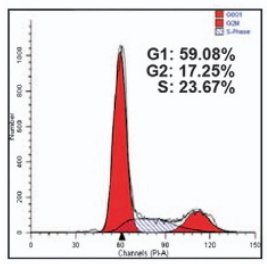

si-NC

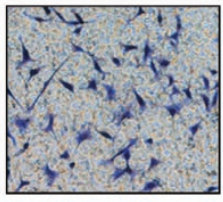

si-CCAT1-2

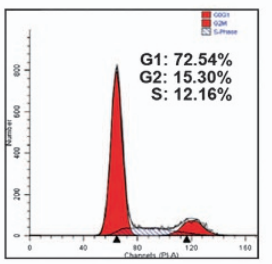

\section{政}
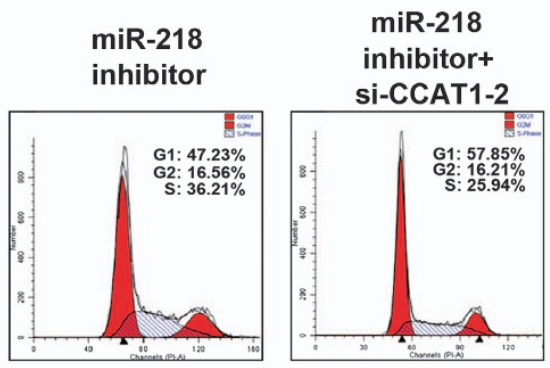

b

b n.s

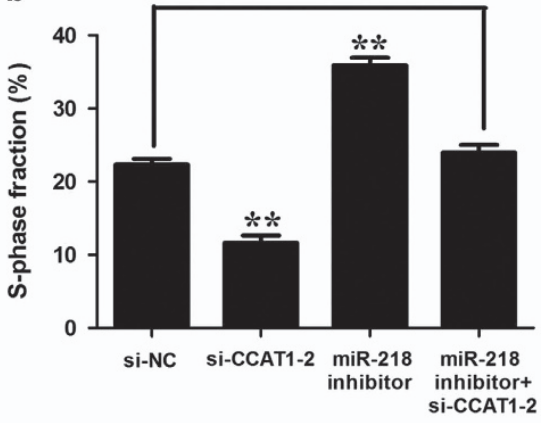

d

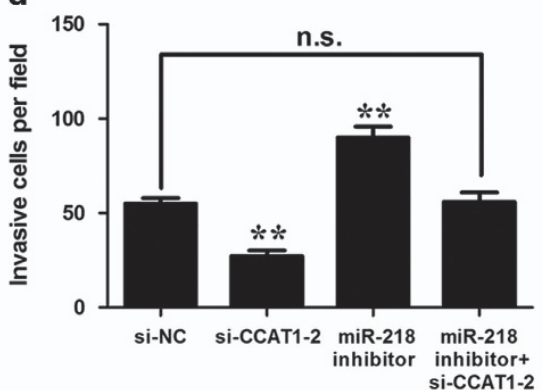

Figure 7 CCAT1's oncogenic activity is in part through negative regulation of miR-218 and then modulating Bmi1. (a) Flow cytometric analysis was performed. Data were expressed as percentage distribution of cells in G0/G1, S and G2/M phases of the cell cycle. (b) Si-CCAT1 induced a reduction of S-phase fraction cells in NOZ cells, which can be rescued by miRNA-218 inhibitor. miRNA-218 inhibitor alone increased the percentage of S-phase cells. (c) Forty-eight hours after transfection, transwell invasion assay was performed. (d) The invasive ability of NOZ cells can be blocked by CCAT1 downregulation. The si-CCAT1-blocked invasive ability of NOZ cells was rescued by miRNA-218 inhibitor, and miRNA-218 inhibitor alone increased the invasive ability. Error bars represent the mean \pm S.D. of triplicate experiments. ${ }^{*} P<0.05 ;{ }^{*} P<0.01 ;$ n.S., not significant

understanding of the ncRNA interaction regulatory network would definitely advance the research in tumorigenesis of GBC.

\section{Experimental Procedure}

Patient samples. The experiments were undertaken with the understanding and written consent of each subject. The study methodologies conformed to the standard set by the Declaration of Helsinki. This study methodology was approved by the Human Ethics Committee of Xinhua Hospital at Shanghai Jiaotong University (Shanghai, China).

The 40 gallbladder carcinoma tissues and their pair-matched adjacent normal gallbladder tissues in this study (collected postoperatively from April 2007 to May 2009) were obtained from patients who underwent radical resections at Xinhua Hospital (Shanghai Jiaotong University School of Medicine, Shanghai, China). Each sample was snap-frozen in liquid nitrogen and stored at $-80^{\circ} \mathrm{C}$ before RNA isolation and qRT-PCR analysis. On removal of the surgical specimen, research personnel transferred the tissues to the surgical lab immediately. Pathology faculty performed a gross evaluation of the specimen and selected the gallbladder tissues that appeared to be cancerous and tissues that appeared to be normal epithelial tissues. Next, a second level of quality control was performed on adjacent normal tissues. Histological slides were prepared from the section of frozen tissue that was selected as normal tissues. These slides were examined by experienced pathologists to determine whether the benign tissues contained any GBC cells. Benign tissues that contained residual cancer tissues were not included in this study. All patients recruited to this study did not receive any pre-operative treatments. GBC patients were staged according to the TNM staging system (the seventh edition) of the American Joint Committee on Cancer staging system. The data do not contain any information that could identify the patients. Complete clinicopathological follow-up data of the GBC patients from which the specimens were collected were available.

Cell culture. Four human GBC cell lines (GBC-SD, SGC-996, NOZ and EHGB2) were used in this study. GBC-SD and SGC-996 were purchased from Cell Bank of the Chinese Academy of Science (Shanghai, China). NOZ was purchased from the Health Science Research Resources Bank (Osaka, Japan). EH-GB2 was a generous gift from Eastern Hepatobiliary Surgical Hospital and Institute, The Second Military University, Shanghai, China. ${ }^{34}$ The non-tumorigenic human intrahepatic biliary epithelial cell line $\mathrm{H} 69$ was purchased from the Health Science Research Resources Bank. The cell lines were cultured in Dulbecco's modified Eagle's medium (Gibco BRL, Grand Island, NY, USA), containg 10\% fetal bovine serum (FBS, HyClone, Invitrogen, Camarillo, CA, USA), as well as $100 \mathrm{U} / \mathrm{ml}$ penicillin and $100 \mu \mathrm{g} / \mathrm{ml}$ streptomycin (Invitrogen, Carlsbad, CA, USA). Cells were maintained in a humidified incubator at $37^{\circ} \mathrm{C}$ in the presence of $5 \% \mathrm{CO}_{2}$. All cell lines have been passaged for fewer than 6 months.

RNA preparation, RT and qPCR. Total RNA from tissues and cells was extracted using Trizol reagent (Invitrogen). RNA was reversed transcribed into cDNAs using the Primer-Script one step RT-PCR kit (TaKaRa, Dalian, China). The cDNA template was amplified by real-time RT-PCR using the SYBR Premix Dimmer Eraser kit (TaKaRa). Gene expression in each sample was normalized to GADPH expression. The primer sequences used were as follows: for GAPDH, 5'-GTCAACGGATTTGGTCTGTATT-3' (forward) and 5'-AGTCTTCTGGGTGGC AGTGAT-3' (reverse); for CCAT1, 5'-TTTATGCTTGAGCCTTGA-3' (forward) and $5^{\prime}$-CTTGCCTGAAATACTTGC-3' (reverse). Primers used in this study were supplied in the Supplementary Materials. Real-time RT-PCR reactions were performed by the ABI7500 system (Applied Biosystems, Carlsbad, CA, USA). The real-time PCRs were performed in triplicate. The relative expression fold change of mRNAs was calculated by the $2^{-\Delta \Delta C t}$ method. Primers used in this study are listed in Supplementary Table 1.

5'- And 3'-rapid amplification of cDNA ends. We used the 5'- and 3'RACE analyses to determine the transcriptional initiation and termination site of CCAT1 using a SMARTer RACE cDNA Amplification Kit (Clontech, Palo Alto, CA, USA), according to the manufacturer's instructions. The gene-specific primers used for the PCR of the RACE analysis were given at Supplementary Table 1.

Real-time quantitative PCR of mature miRNAs. Primers were designed on the basis of miRNA mature sequence. Total RNA from cells was extracted using Trizol reagent (Invitrogen). RNA was reverse transcribed into cDNAs using PrimeScript miRNA cDNA Synthesis Kit (TakaRa), according to the 
a

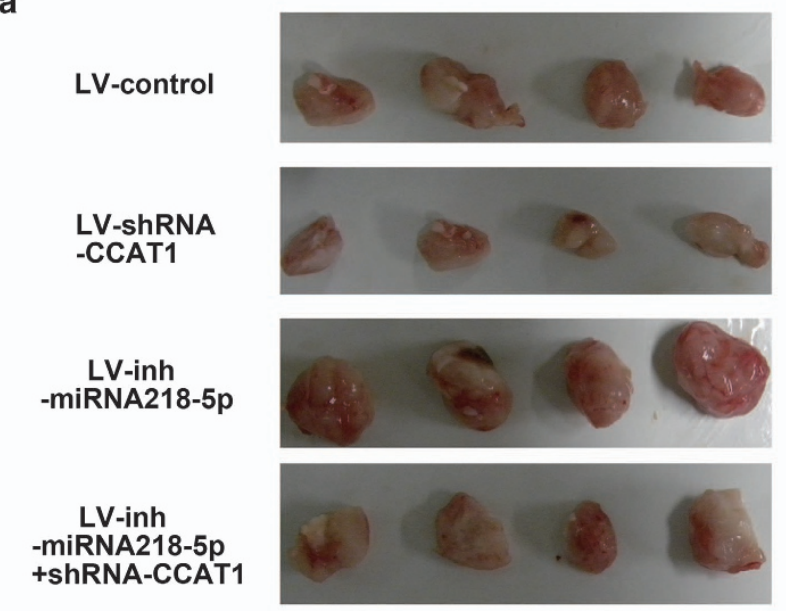

b

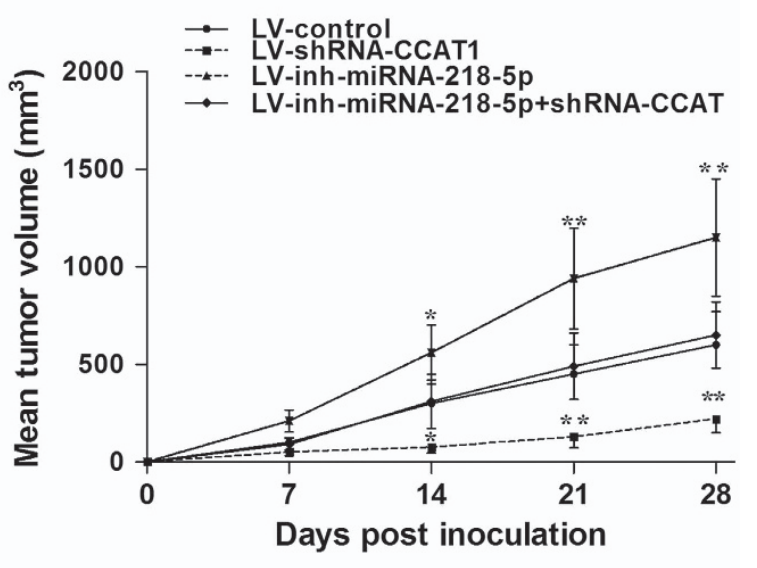

c

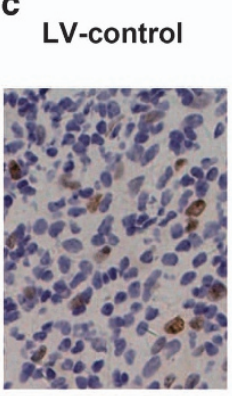

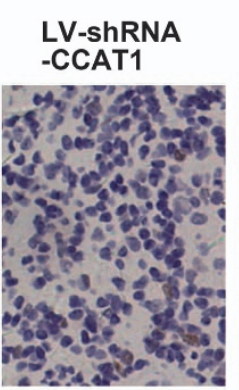

LV-inh -miRNA218-5p
LV-inh -miRNA218-5p +shRNA-CCAT1

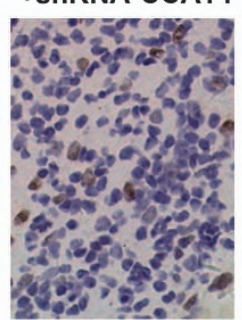

d

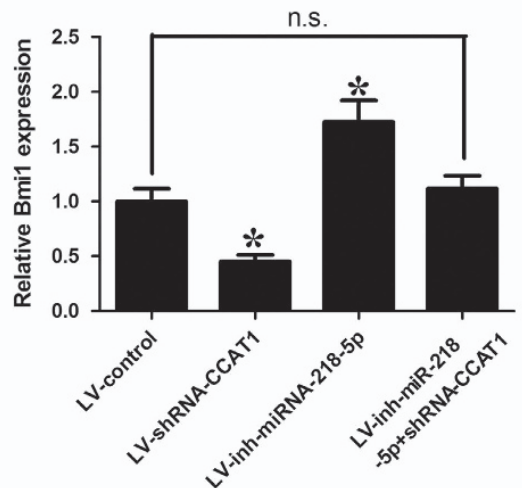

Figure 8 CCAT1's oncogenic activity is in part through negative regulation of miRNA-218-5p in vivo. CCAT1 knockdown suppressed tumor growth in subcutaneous implantation mouse models of NOZ cells and miRNA-218-5p inhibitor abrogated this suppression in tumor growth. Tumor volumes (a) and tumor growth curves (b) of subcutaneous implantation models of GBC are shown. (c, d) Immunohistochemical staining of Bmi1 demonstrated that CCAT1 silencing inhibited the aggressive phenotype of gallbladder cells in vivo, as indicated by the expression of Bmi1-positive cells and miRNA-218-5p inhibitor abolished the inhibition. ${ }^{*} P<0.05,{ }^{* \star} P<0.01$

manufacturer's instructions. The poly (A) was added to the $3^{\prime}$-end of miRNAs. A primer consisting of an oligo(dT) sequence is used for reverse transcription. The qRT-PCR reaction was conducted under the following conditions: single cycle of $300 \mathrm{~s}$ at $95^{\circ} \mathrm{C}, 40$ cycles of $30 \mathrm{~s}$ at $95^{\circ} \mathrm{C}$, followed by $20 \mathrm{~s}$ at $60^{\circ} \mathrm{C}$ and $15 \mathrm{~s}$ at $72{ }^{\circ} \mathrm{C}$. Small nuclear RNA U6 was used as internal control. The real-time PCRs were performed in triplicate. The relative expression fold change of mRNAs was calculated by the $2^{-\Delta \Delta C t}$ method.

Cell transfection. Hsa-miRNA-218-5p mimic/negative control mimic and hsamiRNA-218-5p inhibitor/negative control inhibitor were purchased from Genechem, Shanghai, China. The siRNAs specifically targeting CCAT1 were synthesized by Invitrogen. The siRNA sequences for CCAT1 were si-CCAT1-1, 5'-CGGCAGGCAT TAGAGATGAACAGCA-3' and si-CCAT1-2, 5'-CCATTCCATTCATTTCTCTTTCCTA-3'. The siRNAs specifically targeting Bmi1 were synthesized by Invitrogen. The siRNA sequences for Bmi1 were si-Bmi1-1, 5'-GGAGGAACCTTTAAAGGAT-3' and si-Bmi1-2, 5'-CCAGAGAGATGGACTGACA-3'. Transfections were performed using the Lipofectamine 2000 kit (Invitrogen) according to the manufacturer's instructions.

The cDNA encoding IncRNA-CCAT1 was PCR-amplified by the Pfu Ultra II Fusion HS DNA Polymerase (Stratagene, Agilent Technologies, Santa Clara, CA, USA) and subcloned into the Hindll and EcoRI sites of pcDNA3.1 vector (Invitrogen), named pcDNA3.1-CCAT1. The pcDNA3.1-CCAT1 with point mutations in the miRNA-218-5p response elements (seed sequence binding fragment $5^{\prime}$-TCAAATCCAAAGCACA- $3^{\prime}$ changed to $5^{\prime}$-ACGAGATCAGGATGTT-3') was synthesized using a QuikChange Site-Directed Mutagenesis kit (Stratagene) and named pcDNA3.1-CCAT1-mut (miRNA-218-5p).
The 815-nt region at the $3^{\prime}$-end of either CCAT1 or CCAT1-mut (miRNA-218-5p) was amplified using PCR and subcloned into the pmirGLO vector (Promega, Madison, WI, USA) for Luciferase reporter assay using the one-step directed cloning kit (Novoprotein, Shanghai, China). The $3^{\prime}$-UTR of Bmi1 mRNA containing the intact miRNA-218-5p family recognition sequences were PCR-amplified and subcloned into the Sacl and Sall sites of pmirGLO vector. Primers used in this study are listed in Supplementary Table 1.

Cells were grown on six-well plates to $60 \%$ confluency and transfected using Lipofectamine 2000 (Invitrogen), according to the manufacturer's instructions. Fortyeight hours after transfection, cells were collected for qRT-PCR or western blot analyses. The final concentrations of miRNAs/plasmids employed in this study were as the followings: Bmi1 siRNA/negative control siRNA $20 \mathrm{nM} / \mathrm{ml}$, CCAT1 siRNA/ negative control siRNA $20 \mathrm{nM} / \mathrm{ml}$, CCAT1-wt/CCAT1-mut $50 \mathrm{nM} / \mathrm{ml}$, miRNA-218-5p mimic/negative control mimic $100 \mathrm{nM} / \mathrm{ml}$ and miRNA-218-5p inhibitor/negative control inhibitor $200 \mathrm{nM} / \mathrm{ml}$.

Luciferase reporter assay. pmirGLO, pmirGLO-CCAT1 wt or pmirGLOCCAT1-mut (miRNA-218-5p) was cotransfected with miRNA-218-5p mimics or pmiRNA NC into NOZ cells by Lipofectamine-mediated gene transfer. pmirGLO or pmirGLO-Bmi1 was transfected into different NOZ cell clones and GBC-SD cell clones by Lipofectamine-mediated gene transfer. The relative luciferase activity was normalized to Renilla luciferase activity $48 \mathrm{~h}$ after transfection.

RNA pull-down assay. To determine whether CCAT1 is associated with the RISC complex, we performed RNA pull-down assay using synthesized biotinlabeled CCAT1 as a probe and then detected Ago2 from the pellet by western blotting or detected miRNA-218-5p by qRT-PCR. 
RNA pull-down were performed as described previously. ${ }^{11}$ Briefly, the DNA fragment covering has-miRNA-218-5p seed region binding site of CCAT1 was PCRamplified using a T7-containing primer and then cloned into pCR8 (Invitrogen). In addition, IncRNA loc285194 (Liu et al. ${ }^{11}$ ) was also cloned and used in RNA pull-down assay as a positive control. The resultant plasmid DNA was linearized with restriction enzyme Notl. Biotin-labeled RNAs were in vitro transcribed with the Biotin RNA Labeling Mix (Roche Diagnostics, Indianapolis, IN, USA) and T7 RNA polymerase (Roche, Basel, Switzerland), treated with RNase-free DNase I (Roche) and purified with the RNeasy Mini Kit (Qiagen, Inc., Valencia, CA, USA). Cell extract $(2 \mu \mathrm{g})$ was mixed with biotinylated RNA (100 pmol). Washed Streptavidin agarose beads $(100 \mathrm{ml})$ were added to each binding reaction and further incubated at room temperature for $1 \mathrm{~h}$. Beads were washed briefly three times and boiled in SDS buffer, and the retrieved protein was detected by standard western blot technique.

The Ago2 antibodies used for RIP are purchased from Abcam (Cambridge, MA, USA). The co-precipitated RNAs were detected by RT-PCR. Total RNAs and controls were also assayed to demonstrate that the detected signals were from RNAs specifically binding to Ago2.

Western blotting. Western blot analysis to assess Bmi1 and GADPH expression was carried out as described previously. ${ }^{10} \mathrm{GADPH}$ primary antibodies were purchased from Sigma (St. Louis, MO, USA).

Flow cytometric analysis. Cells transfected with desired plasmid or negative control were plated in six-well plates. After $48 \mathrm{~h}$ incubation, the cultures were incubated with propidium iodide for $30 \mathrm{~min}$ in the dark. Cultures were collected and analysed for cell cycle using a flow cytometer (FACSCalibur, BD Biosciences, San Jose, CA, USA) after propidium iodide staining. Data were expressed as percentage distribution of cells in G0/G1, S and G2/M phases of the cell cycle.

Cell invasion assay. For the invasion assays, $48 \mathrm{~h}$ after transfection, $2 \times 10^{4}$ cells in serum-free media were placed into the upper chamber of an insert $(8.0 \mu \mathrm{m}$, Millipore, Temecula, MA, USA). The chamber was precoated with Matrigel (Sigma). The chambers were then incubated for $24 \mathrm{~h}$ in culture medium with $10 \%$ FBS in the bottom chambers before examination. The cells on the upper surface were scraped and washed away, whereas the invaded cells on the lower surface were fixed and stained for $2 \mathrm{~h}$. Finally, invaded cells were counted under a microscope and the relative number was calculated. Experiments were independently repeated in triplicate.

Xenograft mouse model. NOZ cells $\left(1 \times 10^{6}\right)$ stably expressing control shRNA or CCAT1 shRNA, or miRNA-218-5p inhibitor or CCAT1 shRNA+miRNA218-5p inhibitor were subcutaneously injected into either side of flank area of 4-week-old female athymic nude mice ( $n=3$ mice per group). Tumor volumes were measured $\left(0.5 \times\right.$ length $\times$ width $\left.^{2}\right)$ in mice on a weekly basis. After 4 weeks, mice were killed, and tumors were exercised and subjected to immunohistochemical analysis of Bmi1. All animal experiments were performed in animal laboratory center of Xinhua Hospital and in accordance with the Guide for the Care and Use of Laboratory Animals published by the US National Institutes of Health (NIH publication number $85-23$, revised 1996). The study protocol was approved by the Animal Care and Use committee of Xinhua Hospital (approval ID: 2014041).

Statistics. All statistical analyses were performed using SPSS 17.0 (SPSS, Chicago, IL, USA). The expression differences between GBC and matched normal tissues were analyzed using paired samples $t$-test. Pearson's coefficient correlation was used for expression correlation assay. The expression differences between high/low stages, the expression changes after transfection, S-phase fraction and invasion assay were analyzed using independent samples $t$-test. $P$-values were two-sided and a value of $<0.05$ was considered to be statistically significant.

\section{Conflict of Interest}

The authors declare no conflict of interest.

Acknowledgements. We thank the Eastern Hepatobiliary Surgical Hospital and Institute, The Second Military University, Shanghai, for their generous help. This work was supported by the National Natural Science Foundation of China (grant number 81272747) and Doctorial innovation fund of Shanghai Jiaotong University School of Medicine.

\section{Author contributions}

MZM conceived the study and participated in its design, and coordinated and helped to draft the manuscript. MZM, BFC, YZ and MZW performed the experiments. MDZ and $Y Y Q$ participated in the design of the study and performed the statistical analysis. MZM, ZWQ and WG wrote the paper. All authors read and approved the final manuscript. The funding sources had no role in the study design, in the collection, analysis and interpretation of data, in the writing of the manuscript and in the decision to submit the manuscript for publication.

1. Zhu AX, Hong TS, Hezel AF, Kooby DA. Current management of gallbladder carcinoma. Oncologist 2010; 15: 168-181.

2. Jiao Y, Pawlik TM, Anders RA, Selaru FM, Streppel MM, Lucas DJ et al. Exome sequencing identifies frequent inactivating mutations in BAP1, ARID1A and PBRM1 in intrahepatic cholangiocarcinomas. Nat Genet 2013; 45: 1470-1473.

3. Ooi A, Suzuki S, Nakazawa K, Itakura J, Imoto I, Nakamura $\mathrm{H}$ et al. Gene amplification of Myc and its coamplification with ERBB2 and EGFR in gallbladder adenocarcinoma. Anticancer Res 2009; 29: 19-26.

4. Shi $X$, Sun M, Liu H, Yao Y, Song Y. Long non-coding RNAs: a new frontier in the study of human diseases. Cancer Lett 2013; 339: 159-166.

5. Peng HH, Zhang YD, Gong LS, Liu WD, Zhang Y. Increased expression of microRNA-335 predicts a favorable prognosis in primary gallbladder carcinoma. Onco Targets Ther 2013; 6: 1625-1630.

6. Yeh YM, Chuang CM, Chao KC, Wang LH. MicroRNA-138 suppresses ovarian cancer cell invasion and metastasis by targeting SOX4 and HIF-1 $\alpha$. Int $J$ Cancer 2013; 133: 867-878.

7. Kono H, Nakamura M, Ohtsuka T, Nagayoshi Y, Mori Y, Takahata S et al. High expression of microRNA-155 is associated with the aggressive malignant behavior of gallbladder carcinoma. Oncol Rep 2013; 30: 17-24.

8. Yang F, Xue X, Bi J, Zheng L, Zhi K, Gu Y et al. Long noncoding RNA CCAT1, which could be activated by c-Myc, promotes the progression of gastric carcinoma. J Cancer Res Clin Oncol 2013; 139: 437-445

9. Zhang EB, Yin DD, Sun M, Kong R, Liu XH, You LH et al. P53-regulated long non-coding RNA TUG1 affects cell proliferation in human non-small cell lung cancer, partly through epigenetically regulating HOXB7 expression. Cell Death Dis 2014; 5: e1243.

10. Yang F, Huo XS, Yuan SX, Zhang L, Zhou WP, Wang F et al. Repression of the long noncoding RNA-LET by histone deacetylase 3 contributes to hypoxia-mediated metastasis. Mol Cell 2013; 49: 1083-1096.

11. Liu Q, Huang J, Zhou N, Zhang Z, Zhang A, Lu Z et al. LncRNA loc285194 is a p53-regulated tumor suppressor. Nuclei Acids Res 2013; 41: 4976-4987.

12. Zhang Z, Zhu Z, Watabe K, Zhang X, Bai C, Xu M et al. Negative regulation of IncRNA GAS5 by miR-21. Cell Death Differ 2013; 20: 1558-1568.

13. Jalali S, Bhartiya D, Lalwani MK, Sivasubbu S, Scaria V. Systematic transcriptome wide analysis of IncRNA-miRNA interactions. PLoS One 2013; 8: e53823.

14. Juan L, Wang G, Radovich M, Schneider BP, Clare SE, Wang Y et al. Potential roles of microRNAs in regulating long intergenic noncoding RNAs. BMC Med Genomics 2013; 6 (Suppl 1): S7.

15. Nissan A, Stojadinovic A, Mitrani-Rosenbaum S, Halle D, Grinbaum R, Roistacher M et al. Colon cancer associated transcript-1: a novel RNA expressed in malignant and premalignant human tissues. Int J Cancer 2012; 130: 1598-1606.

16. Karreth FA, Pandolfi PP. ceRNA cross-talk in cancer: when ce-bling rivalries go awry. Cancer Discov 2013; 3: 1113-1121.

17. Huang JF, Guo YJ, Zhao CX, Yuan SX, Wang Y, Tang GN et al. Hepatitis B virus $X$ protein $(\mathrm{HBx})$-related long noncoding RNA (IncRNA) down-regulated expression by HBx (Dreh) inhibits hepatocellular carcinoma metastasis by targeting the intermediate filament protein vimentin. Hepatology 2013; 57: 1882-1892.

18. Li JH, Liu S, Zhou H, Qu LH, Yang JH. starBase v2.0: decoding miRNA-ceRNA, miRNAncRNA and protein-RNA interaction networks from large-scale CLIP-Seq data. Nucleic Acids Res 2014; 42(Database issue): D92-D97.

19. Wang K, Sun T, Li N, Wang Y, Wang JX, Zhou LY et al. MDRL IncRNA regulates the processing of miR-484 primary transcript by targeting miR-361. PLoS Genet 2014; 10: e1004467.

20. Carthew RW, Sontheimer EJ. Origins and mechanisms of miRNAs and siRNAs. Cell 2009; 136: $642-655$.

21. Gregory RI, Chendrimada TP, Cooch N, Shiekhattar R. Human RISC couples microRNA biogenesis and posttranscriptional gene silencing. Cell 2005; 123: 631-640.

22. Karginov FV, Conaco C, Xuan Z, Schmidt BH, Parker JS, Mandel G et al. A biochemical approach to identifying microRNA targets. Proc Natl Acad Sci USA 2007; 104: 19291-19296.

23. Kam Y, Rubinstein A, Naik S, Djavsarov I, Halle D, Ariel I et al. Detection of a long non-coding RNA (CCAT1) in living cells and human adenocarcinoma of colon tissues using FIT-PNA molecular beacons. Cancer Lett 2014; 352: 90-96. 
24. Mathew LK, Skuli N, Mucaj V, Lee SS, Zinn PO, Sathyan P et al. miR-218 opposes a critical RTK-HIF pathway in mesenchymal glioblastoma. Proc Natl Acad Sci USA 2014; 111: 291-296.

25. Zhu Z, Xu Y, Du J, Tan J, Jiao H. Expression of microRNA-218 in human pancreatic ductal adenocarcinoma and its correlation with tumor progression and patient survival. $J$ Surg Oncol 2014; 109: 89-94

26. Yamasaki T, Seki N, Yoshino H, Itesako T, Hidaka H, Yamada Y et al. MicroRNA-218 inhibits cell migration and invasion in renal cell carcinoma through targeting caveolin-2 involved in focal adhesion pathway. J Urol 2013; 190: 1059-1068.

27. Tu Y, Gao X, Li G, Fu H, Cui D, Liu H et al. MicroRNA-218 inhibits glioma invasion, migration, proliferation, and cancer stem-like cell self-renewal by targeting the polycomb group gene Bmi1. Cancer Res 2013; 73: 6046-6055

28. Salmena L, Poliseno L, Tay Y, Kats L, Pandolfi PP. A ceRNA hypothesis: the Rosetta Stone of a hidden RNA language? Cell 2011; 146: 353-358.

29. Wang J, Liu X, Wu H, Ni P, Gu Z, Qiao Y et al. CREB up-regulates long non-coding RNA, HULC expression through interaction with microRNA-372 in liver cancer. Nucleic Acids Res 2010; 38: 5366-5383.

30. Kallen AN, Zhou XB, Xu J, Qiao C, Ma J, Yan L et al. The imprinted H19 IncRNA antagonizes let-7 microRNAs. Mol Cell 2013; 52: 101-112.

31. Han Y, Liu Y, Zhang H, Wang T, Diao R, Jiang Z et al. Hsa-miR-125b suppresses bladder cancer development by down-regulating oncogene SIRT7 and oncogenic long non-coding RNA MALAT1. FEBS Lett 2013; 587: 3875-3882.
32. Cesana M, Cacchiarelli D, Legnini I, Santini T, Sthandier O, Chinappi M et al. A long noncoding RNA controls muscle differentiation by functioning as a competing endogenous RNA. Cell 2011; 147: 358-369.

33. Wang K, Liu F, Zhou LY, Long B, Yuan SM, Wang Y et al. The long noncoding RNA CHRF regulates cardiac hypertrophy by targeting miR-489. Circ Res 2014; 114: 1377-1388.

34. Wang JH, Li LF, Yu Y, Li B, Jin HJ, Shen DH et al. Establishment and characterization of a cell line, EH-GB2, derived from hepatic metastasis of gallbladder cancer. Oncol Rep 2012; 27: 775-782.

(i) Cell Death and Disease is an open-access journal published by Nature Publishing Group. This work is licensed under a Creative Commons Attribution 4.0 International Licence. The images or other third party material in this article are included in the article's Creative Commons licence, unless indicated otherwise in the credit line; if the material is not included under the Creative Commons licence, users will need to obtain permission from the licence holder to reproduce the material. To view a copy of this licence, visit http://creativecommons.org/licenses/by/4.0

Supplementary Information accompanies this paper on Cell Death and Disease website (http://www.nature.com/cddis) 